\title{
Altered gene and protein expression in liver of the obese spontaneously hypertensive/NDmcr-cp rat
}

Jie Chang ${ }^{1,2}$, Shinji Oikawa ${ }^{3}$, Gaku Ichihara ${ }^{2}$, Yui Nanpei ${ }^{4}$, Yasuhiro Hotta ${ }^{4}$, Yoshiji Yamada $^{4}$, Saeko Tada-Oikawa', Hitoshi Iwahashi ${ }^{5,8}$, Emiko Kitagawa $^{5,9}$, Ichiro Takeuchi ${ }^{6}$, Masao Yuda ${ }^{7}$ and Sahoko Ichihara ${ }^{1,4^{*}}$

\begin{abstract}
Background: It is difficult to study the mechanisms of the metabolic syndrome in humans due to the heterogeneous genetic background and lifestyle. The present study investigated changes in the gene and protein profiles in an animal model of the metabolic syndrome to identify the molecular targets associated with the pathogenesis and progression of obesity related to the metabolic syndrome.

Methods: We extracted mRNAs and proteins from the liver tissues of 6- and 25-week-old spontaneously hypertensive/NIH -corpulent rat SHR/NDmcr-cP (CP), SHR/Lean (Lean) and Wistar Kyoto rats (WKY) and performed microarray analysis and two-dimensional difference in gel electrophoresis (2D-DIGE) linked to a matrix-assisted laser desorption ionization time-of-flight tandem mass spectrometry (MALDI-TOF/TOF MS).

Results: The microarray analysis identified 25 significantly up-regulated genes $\left(P<0.01 ; \log _{10}>1\right)$ and 31 significantly down-regulated genes $\left(P<0.01 ; \log _{10}<-1\right)$ in 6 - and 25 -week-old CP compared with WKY and Lean. Several of these genes are known to be involved in important biological processes such as electron transporter activity, electron transport, lipid metabolism, ion transport, transferase, and ion channel activity. MALDI-TOF/TOF MS identified 31 proteins with \pm 1.2 fold change $(P<0.05)$ in 6 - and 25 -week-old $C P$, compared with age-matched WKY and Lean. The up-regulated proteins are involved in metabolic processes, biological regulation, catalytic activity, and binding, while the down-regulated proteins are involved in endoplasmic reticulum stress-related unfolded protein response.

Conclusion: Genes with significant changes in their expression in transcriptomic analysis matched very few of the proteins identified in proteomics analysis. However, annotated functional classifications might provide an important reference resource to understand the pathogenesis of obesity associated with the metabolic syndrome.
\end{abstract}

Keywords: Metabolic syndrome, Pathophysiology, Microarray analysis, Proteomics analysis, Obesity, Liver

\section{Background}

The World Health Organization (WHO) has reported that once considered a problem only in high income countries, overweight and obesity are now dramatically on the rise in low- and middle-income countries, particularly in urban settings [1]. Overweight and obesity related to the metabolic syndrome are also major risk factors for a number of

\footnotetext{
* Correspondence: saho@gene.mie-u.ac.jp

${ }^{1}$ Graduate School of Regional Innovation Studies, Mie University, 1577

Kurimamachiya-cho, Tsu 514-8507, Japan

${ }^{4}$ Department of Human Functional Genomics, Life Science Research Center,

Mie University, Tsu, Japan

Full list of author information is available at the end of the article
}

chronic diseases, including diabetes, cardiovascular diseases, and cancer [2,3]. The metabolic syndrome is defined as a cluster of abdominal obesity, atherogenic dyslipidemia (hypertriglyceridemia, hypo-HDL cholesterolemia, both foster plaque buildup in arterial walls), hypertension, and hyperglycemia (the body cannot properly use insulin or blood sugar) [4]. The etiology of the metabolic syndrome is complex, and is determined by the interplay of both genetic and environmental factors [5]. Several environmental factors, including smoking, highcalorie diet, and physical inactivity influence the development of the metabolic syndrome [6].

\section{Biomed Central}


The spontaneously hypertensive/NIH -corpulent rat (SHR/NDmcr-cp) is a new genetically obese strain that spontaneously develops hypertension, hyperlipidemia, and non-insulin-independent diabetes mellitus [7]. The SHR/NDmcr-cp is a sub-strain of the SHR/N-cp. It has a genetic background from the spontaneously hypertensive rat (SHR) and carries nonsense mutation of leptin receptor derived from obese Koletsky rat [8]. Since the phenotype of the SHR/NDmcr-cp is similar to that of patients with the metabolic syndrome, the SHR/NDmcr$\mathrm{cp}$ is thought to be one of the most suitable animal models of the metabolic syndrome [9]. Given that studying SHR/NDmcr-cp would help to clarify some of the pathophysiological mechanisms of this syndrome, we used this rat model of the metabolic syndrome in the present study.

Using microarray and proteomics analyses, the present study was designed to identify genes and proteins in the liver tissues with altered expression in the SHR/NDmcr$\mathrm{cp}$ rats and to find possible molecular targets associated with the pathogenesis or progression of obesity related to the metabolic syndrome.

\section{Methods}

Animals

SHR/NDmcr-cp (cp/cp) (CP), SHR/Lean (Lean), and Wistar Kyoto rats (WKY) were purchased from the Disease Model Cooperative Research Association (Kyoto, Japan) and used for this study at 6- and 25-weeks of age. All animals were fed normal diet and housed in a temperature-controlled environment $\left(25^{\circ} \mathrm{C}\right)$ with a 12 hour light-dark cycle. The investigation conformed to the Guide for the Care and Use of Laboratory Animals published by the US National Institutes of Health (NIH Publication No. 85-23, revised 1996) and was approved by the Committee on Laboratory Animals Utilization of Mie University.

\section{Measurement of blood pressure and biochemical tests}

Systolic blood pressure (SBP) was measured in six conscious rats per group by the tail-cuff method as described previously [10]. Blood was collected from anesthetized rats, transferred to a chilled tube containing heparin, and centrifuged. Plasma was stored at $-80^{\circ} \mathrm{C}$ until analysis. Serum levels of triglyceride, totalcholesterol, and glucose were measured at SRL (Tokyo, Japan) ( $n=6$ for 6 - and 25-week-old rats).

\section{Preparation of RNA and microarray analysis}

The liver tissues were obtained from 6- and 25-week-old rats $(n=6$, each), immediately frozen in liquid nitrogen, and stored at $-80^{\circ} \mathrm{C}$. Total RNA was extracted from about $250 \mathrm{mg}$ of liver tissue using the RNeasy Mini Kit (Qiagen, Valencia, CA) according to the instructions provided by the manufacturer. Total RNA concentration was quantified by spectrophotometry (ND-1000, NanoDrop Technologies, Wilmington, DE). Then, a search for genome-wide expression changes was conducted using oligo nucleotide microarray (Whole Rat Genome microarray 22 K, Agilent Technologies, Santa Clara, CA) in 1) WKY and CP at 6 weeks of age, 2) Lean and CP at 6 weeks of age, 3) WKY and CP at 25 weeks of age, and 4) Lean and CP at 25 weeks of age. Two microarray slides were used for each comparison in two different RNA samples from each strain.

\section{Hybridization and microarray scanning}

Reverse transcription labeling and hybridization were conducted using the Agilent 60-mer Oligo microarray (Agilent Technologies), according to the instructions provided by the manufacturer. Briefly, total RNA of $500 \mathrm{ng}$ prepared from the individual rat liver tissue samples was used for cDNA synthesis using a T7 promoter primer with Agilent low RNA input fluorescent linear amplification kit. The cRNA was synthesized using T7 RNA polymerase. The reaction was carried out in a solution containing $50 \mathrm{mM} \mathrm{dATP} / \mathrm{dGTP} / \mathrm{dTTP}, 25 \mathrm{mM}$ dCTP, 25 mM cyanine 3 (Cy3)-dCTP (for WKY or Lean sample) or cyanine 5 (Cy5)-dCTP (for CP sample) (NEL580 and 581, Perkin Elmer Life Science, Waltham, MA) and $400 \mathrm{U}$ MMLV reverse transcriptase at $42^{\circ} \mathrm{C}$ for $1 \mathrm{hr}$. The labeled cRNA samples were purified using Qiagen's RNA mini spin columns. Every cyanine-labeled cRNA $(0.75 \mu \mathrm{g})$ was individually hybridized to a rat Agilent Oligo $22 \mathrm{k}$ microarray slide. Hybridization was carried out in $22 \mathrm{~mL}$ of a hybridization mixture containing cDNA probes at $65^{\circ} \mathrm{C}$ for $17 \mathrm{hr}$. The glass slides were then washed with $0: 5 \times$ SSC and $0.01 \%$ sodium dodecyl sulfate (SDS) at room temperature for $5 \mathrm{~min}$, and with 0:06 $\times$ SSC at room temperature for $2 \mathrm{~min}$. Immediately after removing the wash buffer by centrifugation, the glass slides were scanned with an Agilent DNA MicroArray Scanner (Agilent Technologies) containing a $532 \mathrm{~nm}$ laser for Cy3 measurement and a $635 \mathrm{~nm}$ laser for Cy5 measurement.

\section{Quantitative RT-PCR analysis}

To confirm the results of microarray analysis, total RNA extracted from the liver tissue was subjected to quantitative reverse transcription and polymerase chain reaction (RT-PCR) analysis with primers specific for mRNAs encoding ELOVL family member 6, elongation of long chain fatty acids (Elovl6) and fatty acid synthase (Fasn) ( $n=6$ for 6- and 25-week-old rats). Amplification was carried out in a reaction volume of $25 \mu \mathrm{l}$, containing: $12.5 \mu \mathrm{l}$ of FastStart Universal Probe Master $(2 \times)$ (Roche Applied Science, Mannheim, Germany), 200 nM of each primer, $100 \mathrm{nM}$ of Universal ProbeLibrary probe (Roche 
Applied Science) and $2 \mu$ of DNA template. Thermal cycling was initiated with $2 \mathrm{~min}$ incubation at $50^{\circ} \mathrm{C}$, followed by $10 \mathrm{~min}$ denaturation at $95^{\circ} \mathrm{C}$. Then 40 cycles of $95^{\circ} \mathrm{C}$ for $15 \mathrm{~s}$ and $65^{\circ} \mathrm{C}$ for $60 \mathrm{~s}$ were applied. The mRNA for $\beta$-actin was used as an internal control.

\section{Preparation of protein samples}

Frozen liver tissues were also homogenized in lysis buffer (30 mM Tris- $\mathrm{HCl}, 7 \mathrm{M}$ urea $2 \mathrm{M}$ thiourea, $4 \% \mathrm{w} / \mathrm{v}$ CHAPS, and a protease inhibitor cocktail, $\mathrm{pH}$ 8.5). After incubation for $60 \mathrm{~min}$ on ice, homogenates were centrifuged at 30,000 $\times g$ for $30 \mathrm{~min}$ at $4^{\circ} \mathrm{C}$ and the supernatant was collected. Protein concentration was determined in the supernatant by the Bradford assay (Bio-rad Laboratories, Hercules, CA), using bovine serum albumin as a standard [11].

\section{Two-dimensional fluorescence difference gel electrophoresis (2D-DIGE)}

For 2D-DIGE, $25 \mu \mathrm{g}$ of each sample were labeled with 200 pmol of amine-reactive cyanine dyes, Cy3 or Cy5 developed for fluorescence 2D-DIGE technology (GE Healthcare, Buckinghamshire, UK) $(n=4$ for 6 - and 25 -week-old rats) [12]. Internal pools were generated by combining equal amounts of all samples and were labeled with Cy2. Then, two-dimensional gel electrophoresis (2DE) was performed, as described previously [13]. After 2DE, cyaninelabeled proteins were visualized directly by scanning, using the Typhoon 9400 imager (GE Healthcare) in fluorescence mode. The differential in-gel analysis module of the DeCyder software (GE Healthcare) was used for automatic detection followed by editing of protein spots. The same software was used for abundance measurements for each gel by comparing normalized volume ratios of individual spots from Cy3- or Cy5-labeled samples to corresponding Cy2-signals from the pooled samples (internal standard) [14]. Thereafter, all gel comparisons and initial screening type statistical analyses were performed with the biological variation analysis module.

\section{Protein identification}

After image analysis, gels containing the additional load of unlabeled proteins from the liver tissues were stained with Colloidal Coomassie Brilliant Blue G (GE Healthcare) and matched to the fluorescent 2D-DIGE images. Selected spots were picked and in-gel digestion of protein samples was performed using the protocol described in detail previously $[13,15]$. Mass analysis of peptide mixtures was performed using a matrix-assisted laser desorption ionization time-of-flight tandem mass spectrometry (MALDI-TOF/TOF MS; 4800 Plus MALDI TOF/TOF ${ }^{\mathrm{TM}}$ Analyzer, Applied Biosystems, Foster City, CA) operating in positive-ion reflector mode. The Paragon Method was applied for protein database search, using Protein Pilot software (Applied Biosystems) to identify excised proteins.

\section{Western blot analysis}

To confirm the results of proteomic analysis, western blot analysis was conducted. Samples $(n=4$ in each group) containing $10 \mu \mathrm{g}$ of liver tissue proteins were separated by $12 \%$ SDS-PAGE and electroblotted onto polyvinylidene difluoride (PVDF) membranes. The membranes were incubated with rabbit polyclonal antibodies to mouse 10-formyltetrahydrofolate dehydrogenase (FTHFD) (Abcam, Cambridge, UK) at a 1:10,000 dilution and to human carbonic anhydrase III (CA3) (Abcam) at a 1:1,000 dilution. The formed immunocomplexes were visualized by enhanced chemiluminescence (GE Healthcare) using ChemiDox XRS-J (Bio-rad Laboratories). Mouse anti- $\beta$-actin monoclonal antibody (Sigma, St Louis, MO) at a 1:10,000 dilution was used as a loading control. The density of the bands was quantified by Quantity One v3.0 software (Bio-rad Laboratories). Protein expression levels were normalized relative to the level of $\beta$-actin protein in the same tissue sample.

\section{Statistical analysis}

Microarray data analysis was carried out using GeneSpring ver. 7.3.1 software (Agilent Technology) [16,17]. The detected signals were normalized using GeneSpring normalization algorithms. In each comparison, genes were filtered based on minimum \pm 1.0 of log ratio (WKY vs. CP or Lean vs. $\mathrm{CP}$ ) and $\mathrm{P}<0.01$ using Student's test. Gene ontology (GO) analysis was performed using GeneSpring (Agilent Technology). With regard to the data of proteomics analysis, the differential in-gel analysis module of the DeCyder software (GE Healthcare) was used for automatic detection of protein spots and for abundance measurements of each gel by comparing the normalized volume ratios of individual spots [14]. Two-tailed Student's $t$-test was performed to determine the differences between paired groups (WKY vs. CP or Lean vs. CP) using the biological variation analysis module of DeCyder software (GE Healthcare). Data are presented as mean \pm SEM. Differences among physiological and biochemical parameters and the quantitated data of both RT-PCR and western blot analyses were evaluated for statistical significance by oneway analysis of variance followed by Dunnett's post hoc test. Statistical analyses were performed using the JMP 8.0 software (SAS Institute Inc., Cary, NC). A $P$ value of $<0.05$ was considered statistically significant.

\section{Results}

Changes in body weight, systolic blood pressure, and biochemical parameters

Body weight and liver weight were significantly greater in CP than in WKY and Lean at 6 and 25 weeks of age 
(Table 1). CP and Lean became hypertensive from 6 weeks of age, and there was no significant difference in systolic blood pressure at the two ages between CP and Lean (Table 1). At 6 weeks of age, there was no significant difference in triglyceride and glucose levels among the three groups, however, at 25 weeks of age, these levels were significantly higher in CP than in both WKY and Lean (Table 1). These results indicate the development of early stages of the metabolic syndrome at 6 weeks of age and the chronic stage of the syndrome at 25 weeks of age in CP. There were no significant differences in triglyceride and glucose levels between WKY and Lean at the two ages (Table 1). Therefore, we used WKY and Lean as the controls to identify the possible molecular targets associated with the pathogenesis or progression of obesity associated with the metabolic syndrome.

\section{Gene expression profiles by microarray analysis}

After the experiments of microarray analysis, all hybridization spots on the image were quantified. The data of the fluorescence intensity were converted into $\log _{10}$ values. Genes with significantly different expression levels $(\mathrm{P}<0.01)$, relative to $\mathrm{WKY}$ and Lean, were extracted for further analysis. On the oligoDNA microarray, 253 and 125 genes were significantly upregulated $\left(P<0.01\right.$ and $\left.\log _{10}>1\right)$ in 6-week-old CP compared with age-matched WKY and Lean. Furthermore, 244 and 97 genes were down-regulated $(P<0.01$ and $\left.\log _{10}<-1\right)$ in 6 -week-old CP compared with agematched WKY and Lean. We also found 163 and 149 significantly up-regulated genes and 184 and 162 down-regulated genes in 25-week-old CP compared with 25-week-old WKY and Lean. Among these, 25 genes were significantly up-regulated (Additional file 1: Table S1) and 31 genes were significantly downregulated (Additional file 1: Table S2) in the liver tissues of 6- and 25-week-old CP, compared with agematched WKY and Lean. The results of microarray analysis were confirmed by quantitative RT-PCR analysis. The mRNA levels of Elovl6 and Fasn were greater in of 6- and 25-week-old CP, compared with agematched control (WKY and Lean) (Additional file 2: Figure S1).

\section{Functional categories of genes with altered expression in $\mathrm{CP}$}

To understand their biological roles, the genes with significant changes in expression detected in CP by microarray analysis were assigned to established GO classification by GeneSpring ver. 7.3.1. In GO classification, we found three aberrant GO terms $(p<0.01)$ in upregulated genes, electron transporter activity, electron transport, and lipid metabolism and five GO terms $(p<0.01)$ in down-regulated genes electron transporter activity, electron transport, ion transport, transferase, and ion channel activity (Table 2). For the results of Kyoto Encyclopedia of Genes and Genomes (KEGG) molecular pathway analysis, genes with significant change in expression in CP by microarray analysis were also assigned to KEGG molecular pathway by GeneSpring. In KEGG molecular pathway analysis, 10 or 6 predicted pathways were found in the up-regulated and down-regulated genes, respectively (Table 2). The genes with significant change in expression in CP by microarray analysis were also imported into the PANTHER database. The PANTHER classification system indicated that the up-regulated and down-regulated genes in CP can be classified into eight groups according to their functional properties: 1) lipid metabolism, 2) carbohydrate metabolism, 3) protein metabolism, 4) cellular amino acid metabolism, 5) immune system, 6) cell adhesion, 7) signal transduction, and 8) others (Additional file 1: Table S1 and Additional file 1: Table S2).

\section{Identification of proteins with significant change in expression levels}

To investigate the changes in protein expression levels in the liver, proteins extracted from the liver tissues of 6- and 25-week-old WKY, Lean, and CP were subjected to comparative analysis by 2D-DIGE. From approximately 2000 (2295) spots detected in each gel, 307 protein spots were

Table 1 Body and liver tissue weights, blood pressure, and biochemical data in 6- and 25-week-old rats

\begin{tabular}{|c|c|c|c|c|c|c|}
\hline & \multicolumn{3}{|c|}{6 weeks of age } & \multicolumn{3}{|c|}{25 weeks of age } \\
\hline & $\overline{W K Y}$ & Lean & $\mathrm{CP}$ & $\overline{W K Y}$ & Lean & $\mathrm{CP}$ \\
\hline Body weight (g) & $140.2 \pm 1.1$ & $147.5 \pm 1.2$ & $228.5 \pm 1.3^{*} \dagger$ & $422.8 \pm 5.2$ & $413.9 \pm 4.1$ & $578.4 \pm 6.8^{*}+$ \\
\hline Liver weight (g) & $7.2 \pm 0.9$ & $7.5 \pm 0.6$ & $12.5 \pm 1.1^{*} \dagger$ & $12.8 \pm 1.2$ & $13.9 \pm 1.1$ & $28.4 \pm 2.8^{*} \dagger$ \\
\hline Blood pressure (mmHg) & $101.5 \pm 1.2$ & $179.5 \pm 1.4^{*}$ & $173.3 \pm 1.2^{*}$ & $108.7 \pm 1.8$ & $208.7 \pm 2.1^{*}$ & $191.2 \pm 4.0^{*}$ \\
\hline Triglyceride (mg/dl) & $65.8 \pm 7.7$ & $65.2 \pm 3.7$ & $93.3 \pm 13.1$ & $62.8 \pm 12.5$ & $61.8 \pm 5.5$ & $816.3 \pm 51.6^{*}+$ \\
\hline Non-fasting glucose (mg/dl) & $178.4 \pm 7.9$ & $173.7 \pm 7.6$ & $189.2 \pm 4.0$ & $160.7 \pm 5.9$ & $186.2 \pm 34.5$ & $278.2 \pm 26.7^{*}+$ \\
\hline
\end{tabular}

Data are mean \pm SEM of six rats per group.

${ }^{*} P<0.05$, compared with the corresponding value for WKY;

$+P<0.05$, compared with the corresponding value for Lean.

WKY; Wistar Kyoto rats, Lean; spontaneously hypertensive rats (SHR/lean), CP; SHR/NDmcr-cp (cp/cp). 
Table 2 List of entities identified by GO and the pathways identified by KEGG of significantly up-regulated and down-regulated genes in liver tissues in 6- and 25-week-old CP

\begin{tabular}{|c|c|c|}
\hline GO accession & GO Term & $P$ value \\
\hline \multicolumn{3}{|l|}{ Up-regulation } \\
\hline GO:0005489 & electron transporter activity & 7.31E-06 \\
\hline GO:0006118 & electron transport & 7.42E-06 \\
\hline GO:0006629 & lipid metabolism & 5.07E-05 \\
\hline \multicolumn{3}{|c|}{ Down-regulation } \\
\hline GO:0005489 & electron transporter activity & $1.00 \mathrm{E}-03$ \\
\hline GO:0006118 & electron transport & $1.01 \mathrm{E}-03$ \\
\hline GO:0006811 & ion transport & 1.55E-03 \\
\hline GO:0010740 & transferase & 3.29E-03 \\
\hline GO:0005216 & ion channel activity & 4.86E-03 \\
\hline \multicolumn{2}{|c|}{ Predicted pathway } & $P$ value \\
\hline \multicolumn{3}{|l|}{ Up-regulation } \\
\hline \multicolumn{2}{|l|}{ Carbon fixation } & $2.54 \mathrm{E}-06$ \\
\hline \multicolumn{2}{|c|}{ Pentose phosphate pathway } & 1.86E-04 \\
\hline \multicolumn{2}{|c|}{ Pyruvate metabolism } & 4.07E-04 \\
\hline \multicolumn{2}{|c|}{ Glutathione metabolism } & 4.07E-04 \\
\hline \multicolumn{2}{|c|}{ Fructose and mannose metabolism } & 5.27E-04 \\
\hline \multicolumn{2}{|c|}{ Metabolism of xenobiotics by cytochrome P450 } & 7.61E-04 \\
\hline \multicolumn{2}{|c|}{ Fatty acid metabolism } & $9.78 \mathrm{E}-04$ \\
\hline \multicolumn{2}{|c|}{ Glycolysis-Gluconeogenesis } & $1.04 \mathrm{E}-03$ \\
\hline \multicolumn{2}{|c|}{ Arachidonic acid metabolism } & 1.49E-03 \\
\hline \multicolumn{2}{|c|}{ Fatty acid biosynthesis } & 5.44E-03 \\
\hline \multicolumn{3}{|l|}{ Down-regulation } \\
\hline \multicolumn{2}{|c|}{ gamma-hexachlorocyclohexane degradation } & $6.48 \mathrm{E}-07$ \\
\hline \multicolumn{2}{|c|}{ Metabolism of xenobiotics by cytochrome P450 } & 7.14E-06 \\
\hline \multicolumn{2}{|c|}{ Linoleic acid metabolism } & 2.36E-06 \\
\hline \multicolumn{2}{|c|}{ PPARy signaling pathway } & $2.78 \mathrm{E}-03$ \\
\hline \multicolumn{2}{|c|}{ Methionine metabolism } & 7.57E-03 \\
\hline \multicolumn{2}{|c|}{ Glyoxylate and dicarboxylate metabolism } & 7.57E-03 \\
\hline
\end{tabular}

PPARY: peroxisome proliferator-activated receptor $\gamma$.

found to be modified in 6-week-old CP compared with age-matched WKY and Lean, whereas 407 spots were found to be modified in 25-week-old rats. Among these, 63 spots were significantly modified in 6- and 25-week-old $\mathrm{CP}$ according to the DeCyder software analysis with an absolute ratio of more than 1.2-folds $(\mathrm{p}<0.05)$, compared with age-matched WKY and Lean.

These differential spots were cut from the gels, digested and applied to MALDI-TOF-MS analysis. The latter identified 31 spots (Figure 1), which included 13 spots with significantly up-regulated expression in 6- and 25-week-old CP, compared with age-matched WKY and Lean (Table 3), and 18 spots significantly down-regulated in 6- and 25-week-old CP, compared with age-matched WKY and Lean (Table 3). The peptide mass peaks were compared with those in the protein database using Paragon Method. Western blot analysis was also performed to confirm the results of proteomics analysis and the findings (Additional file 2: Figure S2A) were consistent with those of proteomic results; the expression of FTHFD was significantly up-regulated while that of CA3 was significantly down-regulated in 6- and 25-week-old CP compared with age-matched control (WKY and Lean) (Additional file 2: Figure S2B).

\section{Functional categories of protein with altered expression in $\mathrm{CP}$}

To functionally annotate the identified proteins, we mapped them to GO at three levels: cellular component, biological process, and molecular function (Figure 2). The cellular component GO annotation revealed that most of the up-regulated $\mathrm{GO}$ annotated proteins were located at the mitochondrion while half of the downregulated proteins were located at the endoplasmic reticulum (ER) (Figure 2A). The GO annotation for biological process showed that the up-regulated proteins were mainly involved in metabolic processes (Figure 2B). Furthermore, mapping for the molecular function showed that half of the up-regulated proteins belonged to catalytic activity and more than half of the downregulated proteins belonged to binding (Figure 2C).

To understand their biological roles, proteins with significant changes in their expression by proteomics analysis were also imported into the PANTHER database. The PANTHER classification system indicated that the upregulated and down-regulated genes in CP can be classified into six groups according to their functional properties: 1) lipid metabolism, 2) carbohydrate metabolism, 3) protein metabolism, 4) Nucleic acid metabolism, 5) immune system process, and 6) others (Additional file 1: Table S3). Additional file 1: Table S3 also shows the log ratio change of gene expression determined by microarray analysis for proteins identified by proteomics analysis in 6- and 25-week-old CP and age-matched control (WKY and Lean) rats.

\section{Discussion}

Microarray analysis is a powerful tool for the evaluation of expression of many genes in various experimental systems and is particularly suited for the identification of target genes for transcription factors [18]. In the present study, we investigated simultaneous changes in gene and protein profiles using the metabolic syndrome rat model to identify the potential molecular targets associated with obesity- metabolic syndrome. The results of microarray and 2D-DIGE linked to MALDITOF-TOF analyses showed differential mRNA and 

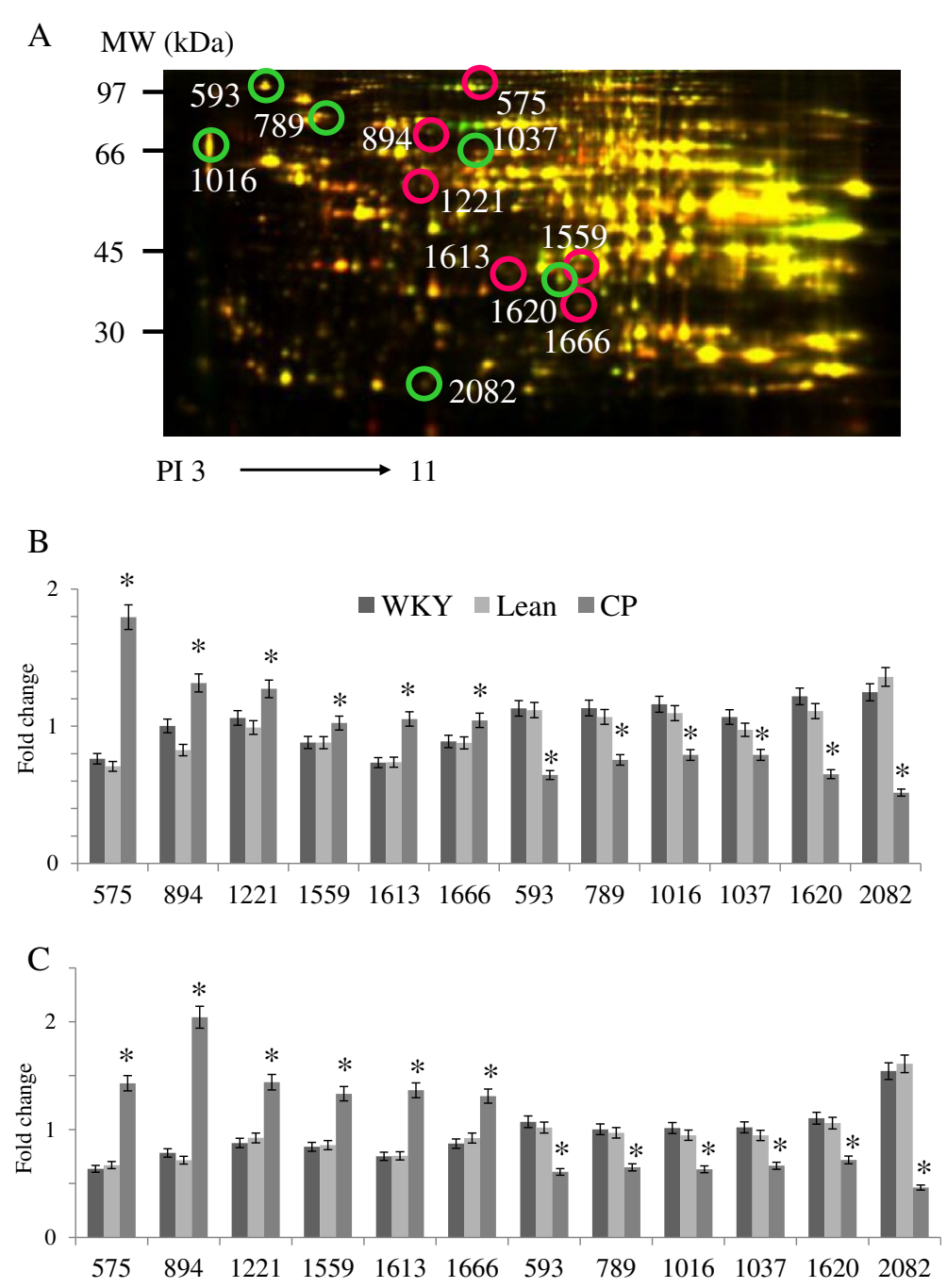

Figure 1 A representative 2D-DIGE image of liver lysates from 25-week-old Lean and CP. (A) The proteins (40 $\mu \mathrm{g}$ each) were labeled with Cy3 and Cy5 dyes, mixed and subjected to 2D-DIGE analysis. We selected significantly different spots of 6- and 25-week-old CP, relative to age-matched control (WKY and Lean) rats. The representative 12 quantified expression levels of each strain at 6 (B) and 25 weeks of age (C) were expressed as mean \pm SEM from four rats per group. ${ }^{*} P<0.05$, compared with the corresponding value for WKY or Lean.

protein expression in the liver tissue in the early and chronic stages of the metabolic syndrome.

In both microarray and proteomics analyses, the only significant change in 6- and 25-week-old CP, compared with the age-matched controls, was in sulfotransferase family $1 \mathrm{C}$, membrane 1 (SULT1C1). SULT1C1 is expressed in the liver, lung, and intestine [19]. One subfamily of this enzyme, estrogen SULT, is expressed in subcutaneous adipose tissue and plays potential roles in glucose homeostasis and inflammation [20,21]. The members of this enzyme are reported to play important roles in not only the metabolism of drugs and xenobiotics but also the biotransformation of a variety of endogenous compounds [22]. Although the enzymatic properties and functional relevance of SULT1C1 remain unknown, SULT1C1 gene expression is reported to be down-regulated in isolated primary hepatocytes from livers of obese insulinresistant Zucker rats [23], consistent with our results. In the present study, only a few of the genes with significant changes in microarray analysis were found to show matching change in the expression of their proteins in proteomics analysis (Tables 3, Additional file 1: Table S1 and Additional file 1: Table S2). Previous studies using high-throughput technologies (transcriptomic and proteomics analyses) demonstrated a positive correlation between transcript and protein levels for the majority of molecules $[24,25]$. Other studies, however, reported limited correlation between transcription and translation in mammals [26]. In the present microarray analysis, we only used genes with significant up-regulation $\left(P<0.01\right.$; $\log _{10}$ $>1)$ or down-regulation $\left(P<0.01 ; \log _{10}<-1\right)$ in 6 - and 25 week-old CP, compared with WKY and Lean. Among the 
Table 3 List of liver tissue proteins with significant spot changes identified in 6- and 25-week-old CP

\begin{tabular}{|c|c|c|c|c|c|c|c|}
\hline \multirow{3}{*}{$\begin{array}{l}\text { Spot } \\
\text { no. }\end{array}$} & \multirow[t]{3}{*}{ Protein name } & \multirow[t]{3}{*}{$\%$ Cover } & \multirow{3}{*}{$\begin{array}{l}\text { Peptides } \\
\text { (95\%) }\end{array}$} & \multicolumn{4}{|c|}{ Fold change } \\
\hline & & & & \multicolumn{2}{|c|}{ 6-week-old } & \multicolumn{2}{|c|}{ 25-week-old } \\
\hline & & & & WKY-CP & Lean-CP & WKY-CP & Lean-CP \\
\hline \multicolumn{8}{|c|}{ Up-regulated proteins } \\
\hline 458 & Pyruvate carboxylase & 3.1 & 3 & 1.15 & 1.15 & 1.27 & 1.22 \\
\hline 561 & Staphylococcal nuclease domain-containing protein 1 & 6.4 & 5 & 1.41 & 1.43 & 2.19 & 2.35 \\
\hline 575 & 10-formyltetrahydrofolate dehydrogenase & 12.4 & 7 & 2.35 & 2.54 & 2.25 & 2.13 \\
\hline 579 & 10-formyltetrahydrofolate dehydrogenase & 10.3 & 5 & 1.91 & 1.89 & 1.96 & 1.84 \\
\hline 595 & Dimethylglycine dehydrogenase & 8.3 & 4 & 1.22 & 1.27 & 1.55 & 1.77 \\
\hline 894 & $\begin{array}{l}\text { Dihydrolipoyllysine-residue acetyltransferase component } \\
\text { of pyruvate dehydrogenase complex }\end{array}$ & 9.7 & 3 & 1.46 & 1.59 & 2.61 & 2.85 \\
\hline 1221 & Glutathione synthetase & 19.2 & 5 & 1.2 & 1.28 & 1.64 & 1.56 \\
\hline 1559 & Glycerol-3-phosphate dehydrogenase [NAD+] & 21 & 6 & 1.16 & 1.16 & 1.77 & 1.54 \\
\hline 1568 & $N(G), N(G)$-dimethylarginine dimethylaminohydrolase 1 & 25.6 & 6 & 1.35 & 1.37 & 2.31 & 1.91 \\
\hline 1604 & Pyruvate dehydrogenase E1 component subunit beta & 25.4 & 7 & 1.18 & 1.25 & 1.6 & 1.68 \\
\hline 1613 & Malate dehydrogenase & 15 & 5 & 1.43 & 1.43 & 1.81 & 1.8 \\
\hline 1666 & Ketohexokinase & 30.2 & 6 & 1.2 & 1.19 & 1.51 & 1.42 \\
\hline 1925 & Glutathione S-transferase kappa 1 & 9.3 & 2 & 1.57 & 1.6 & 1.93 & 1.6 \\
\hline \multicolumn{8}{|c|}{ Down-regulated proteins } \\
\hline 273 & Hypoxia up-regulated protein 1 & 17 & 5 & -1.53 & -1.42 & -1.55 & -1.35 \\
\hline 593 & Endoplasmin & 12.7 & 8 & -1.75 & -1.73 & -1.76 & -1.68 \\
\hline 789 & Protein disulfide-isomerase A4 & 8.6 & 5 & -1.5 & -1.42 & -1.54 & -1.49 \\
\hline 819 & 78 kDa glucose-regulated protein & 14.3 & 6 & -1.39 & -1.27 & -1.69 & -1.47 \\
\hline 820 & 78 kDa glucose-regulated protein & 18.5 & 8 & -1.44 & -1.27 & -1.82 & -1.61 \\
\hline 955 & Alpha-2-HS-glycoprotein & 15.3 & 3 & -1.27 & -1.2 & -2.75 & -3.15 \\
\hline 1016 & Calreticulin & 33.2 & 9 & -1.47 & -1.39 & -1.6 & -1.5 \\
\hline 1037 & Protein disulfide-isomerase $\mathrm{A} 3$ & 19.4 & 6 & -1.31 & -1.23 & -1.53 & -1.42 \\
\hline 1053 & Protein disulfide-isomerase A3 & 14.7 & 5 & -1.41 & -1.28 & -1.52 & -1.48 \\
\hline 1217 & Keratin, type I cytoskeletal 18 & 11.1 & 4 & -1.52 & -1.44 & -1.61 & -1.63 \\
\hline 1499 & Farnesyl pyrophosphate synthase & 9.9 & 3 & -1.24 & -1.22 & -1.83 & -1.76 \\
\hline 1598 & Regucalcin & 24.4 & 7 & -1.27 & -1.19 & -1.7 & -1.85 \\
\hline 1620 & Sulfotransferase $1 \mathrm{C} 1$ & 30.3 & 6 & -1.87 & -1.71 & -1.54 & -1.48 \\
\hline 1840 & C-reactive protein & 8.7 & 6 & -1.23 & -1.23 & -1.89 & -1.7 \\
\hline 1860 & Carbonic anhydrase 3 & 51.5 & 10 & -4.27 & -4.84 & -2.12 & -2.36 \\
\hline 1862 & Carbonic anhydrase 3 & 43.5 & 8 & -2.69 & -3.24 & -1.72 & -1.97 \\
\hline 2082 & Major urinary protein & 37.6 & 6 & -2.42 & -2.64 & -3.34 & -3.48 \\
\hline 2164 & Cytochrome b5 & 55.2 & 7 & -1.18 & -1.18 & -1.68 & -1.52 \\
\hline
\end{tabular}

Abbreviations as in Table 1.

27 identified proteins in proteomics analysis, the gene expression of 15 proteins was up-regulated or downregulated by more than 1.5 fold in 6- and 25-week-old CP, compared with WKY and Lean (Additional file 1: Table S3). The different expression patterns noted in the two methodological approaches might arise from limitation of detection sensitivity or electrophoretic separation. Moreover, the discrepancy between the microarray and proteomics analyses might be due to differential regulation of translation, turnover, or alternative splicing.

The cellular component GO annotation revealed that the up-regulated $\mathrm{GO}$ annotated proteins were located in the mitochondria (90\%) while the down-regulated proteins were mapped to the ER (65\%). Our study showed down-regulation of both CRT and PDIA3/4. CRT is a highly versatile lectin-like chaperone involved in many 


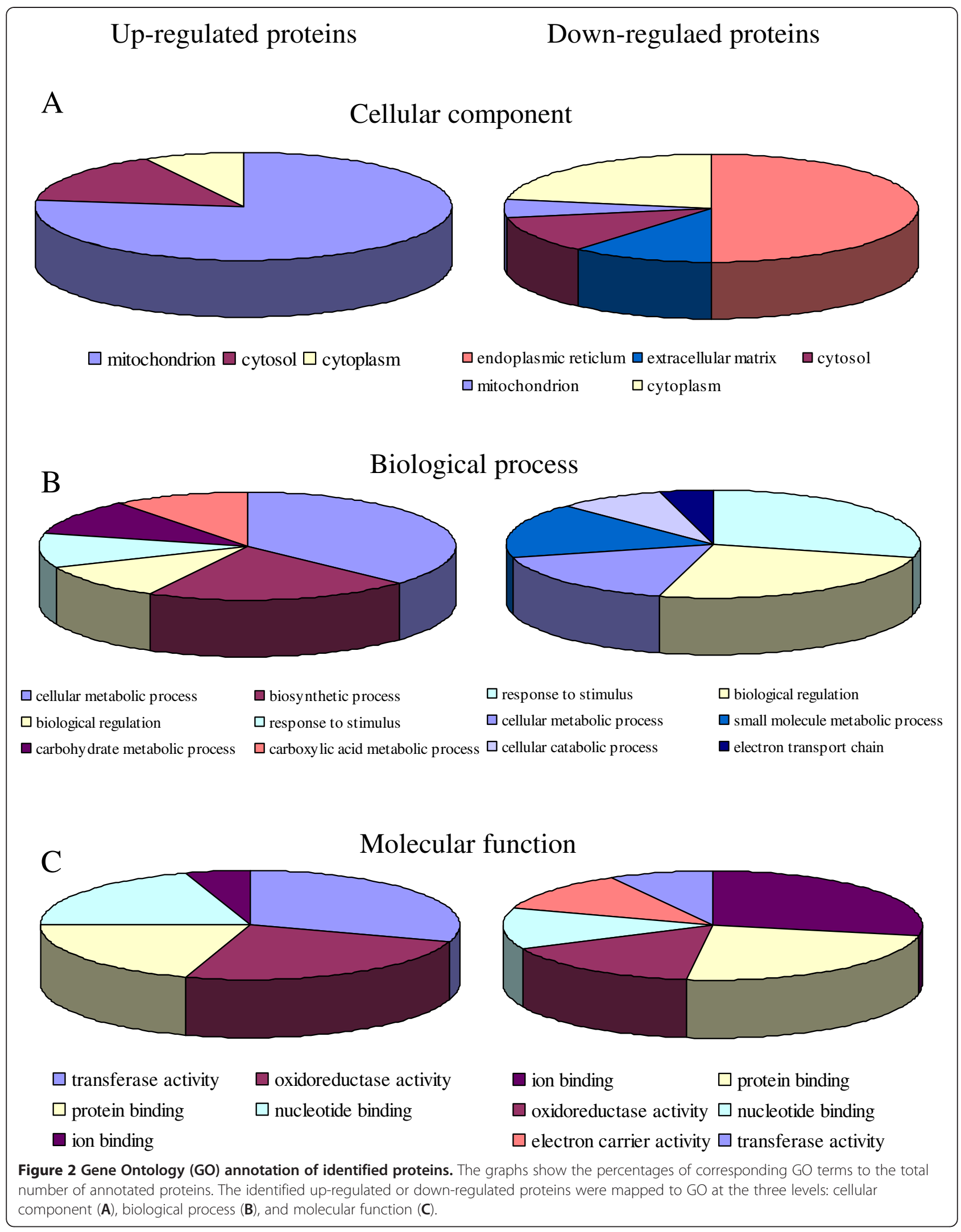


cellular functions both inside and outside ER lumen [27]. The CRT-associated functions include acting as chaperone of nascent glycoproteins, regulator of $\mathrm{Ca}^{2+}$ homeostasis, cell adhesion, and inhibition of angiogenesis and tumor growth [28]. A highly adipogenic signal was detected in embryonic stem cells from Crt-deficient mice [29] and high glucose uptake and glycogen deposition were described in ventricular cardiomyocytes of these mice [30]. Crt deficiency also correlated with significant increases in insulin receptor expression, stability of glucose transporter 1 (GLUT1) expression, and in insulin-stimulated Akt phosphorylation and kinase activity, suggesting that the lack of CRT is associated with changes in insulin signaling and glucose metabolism [31-33]. While the potential effects of PDIA3 and PDIA4 are unknown, oxidative folding of glycoproteins in the ER and/or interaction with calnexin (CNX) and CRT could be affected [34]. PDI acts as a chaperone to promote oxidative refolding of non-monoglycosylated and reduces denatured lysozymes in the absence of CNX/ CRT in vitro [35]. Furthermore, PDI is reported to interact with a specific set of glycoproteins that are recruited via its interactions with the lectins CNX/CRT $[36,37]$. ER stress has recently been implicated in the pathophysiology of obesity-related insulin resistance; however, what causes ER stress in obesity remains uncertain [38]. In our study, significant down-regulation of CRT and PDIA3/4 was noted in CP compared with WKY and Lean. Since the proteins involved in ER stress-related unfolded protein response were down-regulated, they may induce resistance to insulin and play an important role in the development of obesity associated with the metabolic syndrome.

\section{Conclusion}

In the present study, we applied comparative genomic and proteomic analyses to identify the molecular targets in liver tissue associated with obesity of the metabolic syndrome. Microarray analysis identified 25 significantly up-regulated genes and 31 significantly down-regulated genes in the liver tissues of 6- and 25-week-old CP, compared with age-matched WKY and Lean. To gain further insight into the roles of these genes in biological processes, the genes with significantly change in their expression were classified according to their function. A large proportion of the genes were involved in electron transporter activity, electron transport, lipid metabolism, ion transport, transferase, and ion channel activity. Using proteomics approach, the up-regulated proteins were involved in metabolic process, biological regulation, catalytic activity, and binding. On the other hand, the downregulated proteins were involved in ER stress-related unfolded protein response. These findings suggest that both groups of proteins might contribute to the development of obesity associated with the metabolic syndrome. These molecules may provide an important reference resource and be applied to the development of therapeutic targets for obesity associated with the metabolic syndrome.

\section{Additional files}

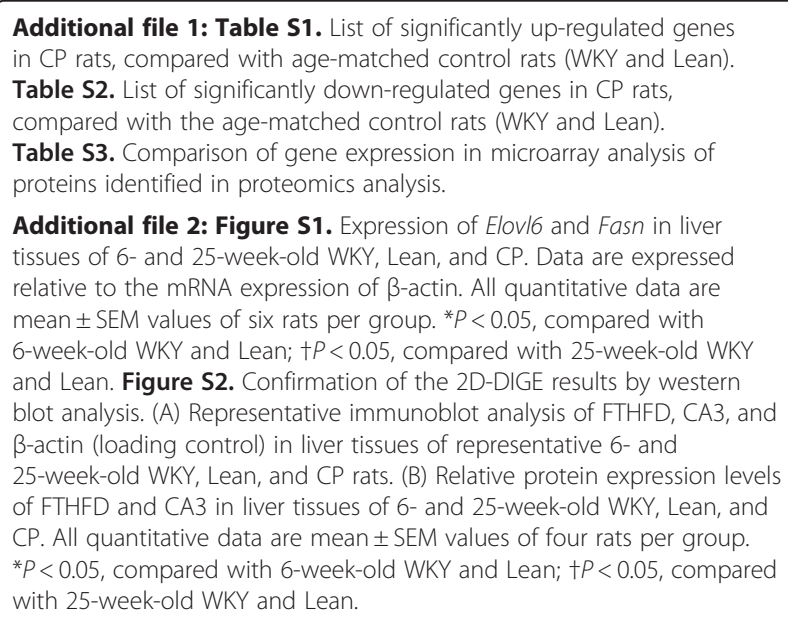

Additional file 2: Figure S1. Expression of Elovl6 and Fasn in liver tissues of 6- and 25-week-old WKY, Lean, and CP. Data are expressed relative to the mRNA expression of $\beta$-actin. All quantitative data are mean \pm SEM values of six rats per group. ${ }^{*} P<0.05$, compared with 6-week-old WKY and Lean; $+P<0.05$, compared with 25-week-old WKY and Lean. Figure S2. Confirmation of the 2D-DIGE results by western blot analysis. (A) Representative immunoblot analysis of FTHFD, CA3, and $\beta$-actin (loading control) in liver tissues of representative 6- and 25-week-old WKY, Lean, and CP rats. (B) Relative protein expression levels of FTHFD and CA3 in liver tissues of 6- and 25-week-old WKY, Lean, and $C P$. All quantitative data are mean \pm SEM values of four rats per group. ${ }^{*} P<0.05$, compared with 6-week-old WKY and Lean; $+P<0.05$, compared with 25-week-old WKY and Lean.

\section{Abbreviations}

2D-DIGE: Two-dimensional fluorescence difference gel electrophoresis; CA3: Carbonic anhydrase III; CNX: Calnexin; CRT: Calreticulin; ELOVL6: ELOVL family member 6, elongation of long chain fatty acids; ER: Endoplasmic reticulum; FASN: Fatty acid synthase; FTHFD: 10-formyltetrahydrofolate dehydrogenase; MALDI-TOF/TOF MS: Matrix-assisted laser desorption ionization time-of-flight tandem mass spectrometry; PDIA A3/4: Protein disulfide-isomerase A3/4; RT-PCR: Quantitative reverse transcription and polymerase chain reaction; SBP: Systolic blood pressure; SHR/NDmcrcp: Spontaneously hypertensive/NIH -corpulent rat; WKY: Wistar Kyoto rats.

\section{Competing interests}

The authors declare no conflict of interest.

\section{Authors' contribution}

JC performed the experiments, analysis, and manuscript writing, SO and Gl contributed to study design and manuscript writing, YN performed the proteomics analysis, YH performed the microarray analysis, YY and ST-O participated in data interpretation of proteomics analysis, HI, EK, IT, and MY participated in data interpretation of microarray analysis, SI participated in organization of the study, data interpretation, and preparation of the manuscript. All authors read and approved the final manuscript.

\section{Acknowledgments}

The authors thank Kiyora Izuoka for assistance with biochemical analysis and Kumi Nakao for help in preparation of the manuscript. This work was supported in part by grants from the Japan Society for the Promotion of Science (grants-in aid for Scientific Research \#22390122 and NEXT Program \#LS056).

\section{Author details}

${ }^{1}$ Graduate School of Regional Innovation Studies, Mie University, 1577 Kurimamachiya-cho, Tsu 514-8507, Japan. ${ }^{2}$ Department of Environmental and Occupational Health, Nagoya University Graduate School of Medicine, Nagoya, Japan. ${ }^{3}$ Department of Molecular and Environmental Medicine, Mie University Graduate School of Medicine, Tsu, Japan. ${ }^{4}$ Department of Human Functional Genomics, Life Science Research Center, Mie University, Tsu, Japan. ${ }^{5}$ Health Technology Research Center, National Institute of Advanced Industrial Science and Technology, Tsukuba, Japan. ${ }^{6}$ Department of Engineering, Nagoya Institute of Technology, Nagoya, Japan. ${ }^{7}$ Department of 
Medical Zoology, Mie University Graduate School of Medicine, Tsu, Japan. ${ }^{8}$ Graduate School of Applied Biological Sciences, Gifu University, Gifu, Japan.

${ }^{9}$ Applied Science, Roche Diagnostics, Tokyo, Japan.

Received: 24 April 2012 Accepted: 7 September 2012

Published: 21 September 2012

\section{References}

1. Fall CH, Borja JB, Osmond C, Richter L, Bhargava SK, Martorell R, Stein AD, Barros FC, Victora CG, COHORTS group: Infant-feeding patterns and cardiovascular risk factors in young adulthood: data from five cohorts in low- and middle-income countries. Int J Epidemiol 2011, 40:47-62.

2. Arnlöv J, Ingelsson E, Sundström J, Lind L: Impact of body mass index and the metabolic syndrome on the risk of cardiovascular disease and death in middle-aged men. Circulation 2010, 121:392-400.

3. Rosato V, Zucchetto A, Bosetti C, Dal Maso L, Montella M, Pelucchi C, Negri E, Franceschi S, La Vecchia C: Metabolic syndrome and endometrial cancer risk. Ann Oncol 2011, 22:884-889.

4. Hsieh SD, Muto T, Tsuji H, Arase Y, Murase T: Clustering of other metabolic risk factors in subjects with metabolic syndrome. Metabolism 2010, 59:697-702.

5. Yamada Y, Ichihara S, Kato K, Yoshida T, Yokoi K, Matsuo H, Watanabe S, Metoki N, Yoshida H, Satoh K, Aoyagi Y, Yasunaga A, Park H, Tanaka M, Lee W, Nozawa Y: Genetic risk for metabolic syndrome: examination of candidate gene polymorphisms related to lipid metabolism in Japanese people. J Med Genet 2008, 45:22-28.

6. Park YW, Zhu S, Palaniappan L, Heshka S, Carnethon MR, Heymsfield SB: The metabolic syndrome: prevalence and associated risk factor findings in the US population from the Third National Health and Nutrition Examination Survey. Arch Intern Med 2003, 163:427-436.

7. Striffler JS, Bhathena SJ, Michaelis OE 4th, Campbell JD, Hansen CT, Scalbert E, Thibault N, Velasquez MT: Long-term effects of perindopril on metabolic parameters and the heart in the spontaneously hypertensive/ $\mathrm{NIH}$-corpulent rat with non-insulin-dependent diabetes mellitus and hypertension. Metabolism 1998, 47:1199-1204.

8. Koletsky S: Obese spontaneously hypertensive rats-a model for study of atherosclerosis. Exp Mol Pathol 1973, 19:53-60.

9. Yamamoto J, Ikena K, Yamori Y: Obese and hypertensive SHR/NDmcr-cp rats -A model of metabolic syndrome. Adiposcience 2005, 2:243-248.

10. Ichihara S, Noda A, Nagata K, Obata K, Xu J, Ichihara G, Oikawa S, Kawanish S, Yamada Y, Yokota M: Pravastatin increases survival and suppresses an increase in myocardial matrix metalloproteinase activity in a rat model of heart failure. Cardiovasc Res 2006, 69:726-735

11. Nishizawa T, Iwase M, Kanazawa H, Ichihara S, Ichihara G, Nagata K, Obata K, Kitaichi K, Yokoi T, Watanabe M, Tsunematsu T, Ishikawa Y, Murohara T, Yokota M: Serial Alteration of $\beta$-adrenergic signaling in dilated cardiomyopathic hamsters. Circ J 2004, 68:1051-1060.

12. Kondo T, Hirohashi S: Application of highly sensitive fluorescent dyes (CyDye DIGE Fluor saturation dyes) to laser microdissection and twodimensional difference gel electrophoresis (2D-DIGE) for cancer proteomics. Nat Protoc 2006, 1:2940-2056.

13. Oikawa S, Yamada T, Minohata T, Kobayashi H, Furukawa A, Tada-Oikawa S, Hiraku Y, Murata M, Kikuchi M, Yamashima T: Proteomic identification of carbonylated proteins in the monkey hippocampus after ischemiareperfusion. Free Radic Biol Med 2009, 46:1472-1477.

14. Furukawa A, Oikawa S, Hasegawa-Ishii S, Chiba Y, Kawamura N, Takei S, Yoshikawa K, Hosokawa M, Kawanishi S, Shimada A: Proteomic analysis of aging brain in SAMP10 mouse: a model of age-related cerebral degeneration. Mech Ageing Dev 2010, 131:379-388.

15. Huang Z, Ichihara S, Oikawa S, Chang J, Zhang L, Takahashi M, Subramanian K, Mohideen SS, Wang Y, Ichihara G: Proteomic analysis of hippocampal proteins of $\mathrm{F} 344$ rats exposed to 1-bromopropane. Toxicol Appl Pharmacol 2011, 257:93-101.

16. Iwahashi H, Kitagawa E, Suzuki Y, Ueda Y, Ishizawa YH, Nobumasa H, Kuboki $Y$, Hosoda $H$, Iwahashi $Y$ : Evaluation of toxicity of the mycotoxin citrinin using yeast ORF DNA microarray and Oligo DNA microarray. BMC Genomics 2007, 8:95.

17. Iwahashi Y, Kitagawa E, Iwahashi H: Analysis of mechanisms of T-2 toxin toxicity using yeast DNA microarrays. Int J Mol Sci 2008, 9:2585-2600

18. Relógio A, Schwager C, Richter A, Ansorge W, Valcárcel J: Optimization of oligonucleotide-based DNA microarrays. Nucleic Acids Res 2002, 30:e51.
19. Sakakibara Y, Yanagisawa K, Katafuchi J, Ringer DP, Takami Y, Nakayama T, Suiko M, Liu M-C: Molecular cloning, expression, and characterization of novel human SULT1C sulfotransferases that catalyze the sulfonation of N-hydroxy-2-acetylaminofluorene. J Biol Chem 1998, 273:33929-33935.

20. Khor VK, Dhir RD, Yin X, Ahima RS, Song W-C: Estrogen sulfotransferase regulates body fat and glucose homeostasis. Am J Physiol Endocrinol Metab 2010, 299:E657-E664.

21. Ahima RS, Stanley TL, Khor VK, Zanni MV, Grinspoon SK: Estrogen sulfotransferase is expressed in subcutaneous adipose tissue of obese humans in associated with TNF-a and SOCS3. I Clin Endocrinol Metab 2011, 96:E1153-E1158.

22. Freimuth RR, Raftogianis RB, Wood TC, Moon E, Kim U-J, Xu J, Siciliano MJ, Weinshilboum RM: Human sulfotransferases SULT1C1 and SULT1C2: CDNA characterization, gene cloning, and chromosomal localization. Genomics 2000, 65:157-165.

23. Buqué X, Martínez MJ, Cano A, Miquilena-Colina ME, García-Monzón C, Aspichueta P, Ochoa B: A subset of dysregulated metabolic and survival genes is associated with severity of hepatic steatosis in obese Zucker rats. J Lipid Res 2010, 51:500-513.

24. Kislinger T, Cox B, Kannan A, Chung C, Hu P, Ignatchenko A, Scott MS, Gramolini AO, Morris Q, Hallett MT, Rossant J, Hughes TR, Frey B, Emili A: Global survey of organ and organelle protein expression in mouse: combined proteomic and transcriptomic profiling. Cell 2006, 125:173-186.

25. Hornshøj H, Bendixen E, Conley LN, Andersen PK, Hedegaard J, Panitz F, Bendixen C: Transcriptomic and proteomic profiling of two porcine tissues using high-throughput technologies. BMC Genomics 2009, 10:30.

26. Rogers S, Girolami M, Kolch W, Waters KM, Liu T, Thrall B, Wiley HS: Investigating the correspondence between transcriptomic and proteomic expression profiles using coupled cluster models. Bioinformatics 2008, 24:2894-2900.

27. Qiu Y, Marek M: Transcriptional control of the calreticulin gene in health and disease. Int I Biochem Cell Biol 2009, 41:531-538.

28. Ferreira V, Molina MC, Valck C, Rojas A, Aguilar L, Ramírez G, Schwaeble W, Ferreira A: Role of calreticulin from parasites in its interaction with vertebrate hosts. Mol Immun 2004, 40:1279-1291.

29. Szabo E, Feng T, Dziak E, Opas M: Cell adhesion and spreading affect adipogenesis from embryonic stem cells. The role of calreticulin. Stem Cells 2009, 27:2092-2102.

30. Lozyk MD, Papp S, Zhang X, Nakamura K, Michalak M, Opas M: Ultrastructural analysis of development of myocardium in calreticulindeficient mice. BMC Dev Biol 2006, 6:54.

31. Bass J, Chiu G, Argon Y, Steiner DF: Folding of insulin receptor monomers is facilitated by the molecular chaperones calnexin and calreticulin and impaired by rapid dimerization. J Cell Biol 1998, 141:637-646.

32. Jain HT, Many TN, Riahi Y, Kaiser N, Eckel J, Sasson S: Calreticulin destabilizes glucose transporter-1 mRNA in vascular endothelial and smooth muscle cells under high-glucose conditions. Cir Res 2005, 97:1001-1008

33. Jalali S, Aghasi M, Yeganeh B, Mesaeli N: Calreticulin regulates insulin receptor expression and its downstream PI3 Kinase/Akt signalling pathway. Biochim Biophy Acta 2008, 1783:2344-2351.

34. Hatahet F, Ruddock LW: Substrate recognition by the protein disulfide isomerases. FEBS J 2007, 274:5223-5234.

35. Puig A, Gilbert HF: Protein disulfide isomerase exhibits chaperone and anti-chaperone activity in the oxidative refolding of lysozyme. J Biol Chem 1994, 269:7764-7771

36. Jessop CE, Chakravarthi S, Garbi N, Hämmerling GJ, Lovell S, Bulleid NJ: ERp57 is essential for efficient folding of glycoproteins sharing common structural domains. EMBO J 2007, 26:28-40,

37. Rutkevich LA, Cohen-Doyle MF, Brockmeier U, Williams DB: Functional relationship between protein disulfide isomerase family members during the oxidative folding of human secretory proteins. Mol Biol Cell 2010, 21:3093-3105.

38. Boden G, Song W, Duan X, Cheung P, Kresge K, Barrero C, Merali S: Infusion of glucose and lipids at physiological rates causes acute endoplasmic reticulum stress in rat liver. Obesity 2011, 19:1366-1373.

doi:10.1186/1743-7075-9-87

Cite this article as: Chang et al:: Altered gene and protein expression in liver of the obese spontaneously hypertensive/NDmcr-cp rat. Nutrition \& Metabolism 2012 9:87. 